\title{
Upper Gastrointestinal Hemorrhage Associated with Fistula Formation in the Duodenum due to a Catheter Placed in the Hepatic Artery
}

\author{
M. Muñoz-Navas, J. Jimenez-Perez, J. Sagues and J. Zozaya \\ Division of Gastroenterology. Clínica Universitaria. Faculty of Medicine. University of \\ Navarra, Pamplona, Spain
}

Various fistulous complications have been described in the gastrointestinal tract associated with the presence of a prosthesis or catheters located in the aorta or in other great abdominal arteries, either to resolve aneurysms or to bypass occlusive diseases. Exceptionally, enteric fistulas associated with a surgically implanted arterial chemotherapy infusion catheter in the hepatic artery may also occur, as has been previously demonstrated $(2,3)$.

This report describes a patient with a hepatic carcinoma treated with arterial chemotherapy, using a polyethylene catheter placed in the hepatic artery through the gastroduodenal artery. Seven months later, the patient returned to our hospital with hematemesis and melena. An esophagogastroduodenoscopy revealed a duodenal ulcer with signs of recent hemorrhage, in which a portion of the thin infusion catheter was seen (Fig. 1). Duodenal lesions secondary to fistulas due to abdominal prosthesis or intravascular catheters must be included among the various causes of upper gastrointestinal bleeding.

The cause of these fistulas appears to be due to contact between a foreign body and the intestinal wall (2). Added to this are the pulsating movements of the arterial prosthesis, intestinal motility and all the various factors which change abdominal pressure, such as respiratory movements and exercise (4). This situation leads to an inflammatory reaction which causes adhesion of the intestinal wall to the foreign body. The consequence is gradual erosion of the intestinal wall (1) and fistula formation. In the case of catheters used for intra-arterial chemotherapy, the decrease in the blood flow in the duodenum after placement of the catheter, and the injury caused in the duodenal mucosa by chemotherapeutic agents have been considered as possible adjuvant factors (2).

\section{REFERENCES}

1. Bussuttil, R.W., W. Rees, J.D. Baker, S.E. Wilson: Pathogenesis of aortoduodenal fistula; experimental and clinical correlates. Surgery 85 (1979) 1

2. Crowley, M.L.: Penetrating duodenal ulcer associated with an operatively implanted arterial chemotherapy infusion catheter. Gastroenterology 83 (1982) 118

3. Gillin, J.S., N. Kemeny, J.M. Daly, M.B. Balis, R.C. Kurtz, M. Shike: Severe gastroduodenal ulcerations complicating hepatic artery infusion chemotherapy for metastatic colon cancer. Gastroenterology 84 (1983) 1166

4. Monson, J.R.T., D.F. Courtney, N.A.G. Jones, R.C. Kester: Cannibalization of a goretex aortohepatic graft by the duodenum. Br. J. Surg. 72 (1985) 101 

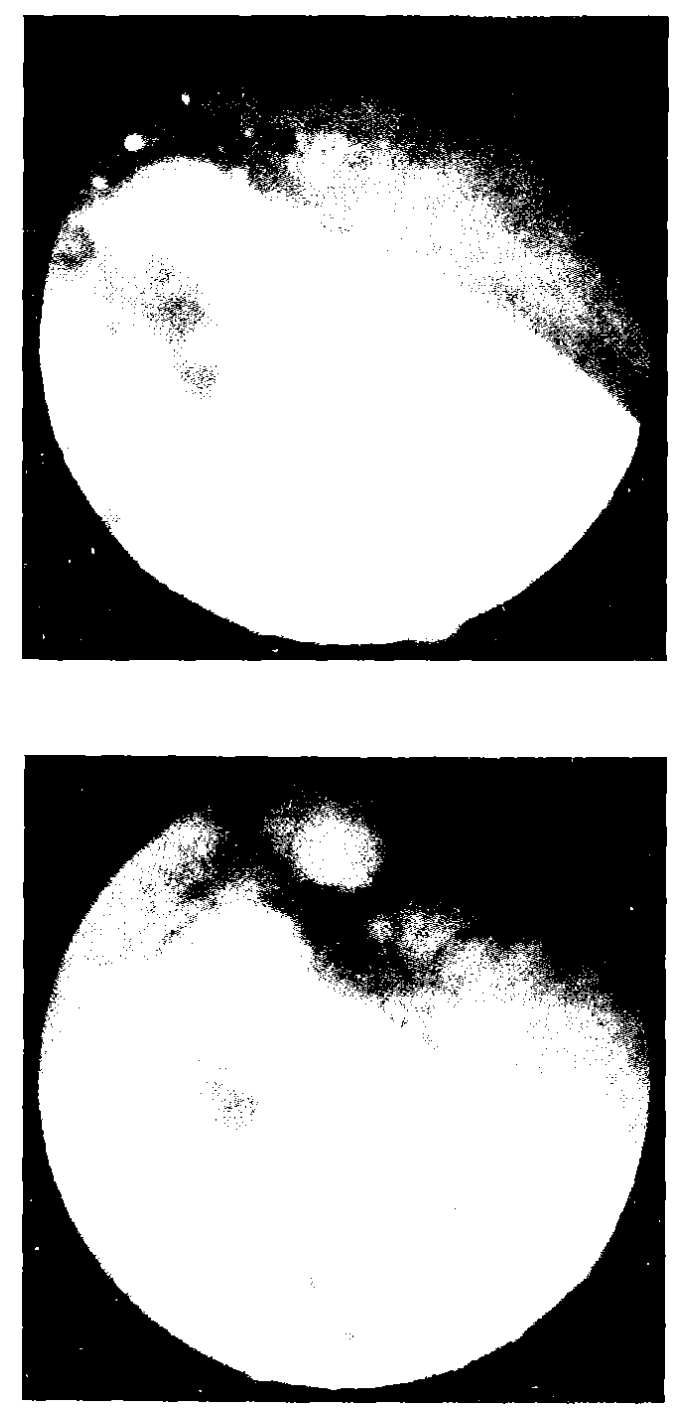

Figure 1. Long shallow duodenal ulcer, with a small clot at one edge, and a portion of the polyethylene catheter running through the ulcer crater from one margin to the other. (The photograph was obtained with an Olympus GIF-XQ endoscope) 